\title{
ESCALA DE AVALIAÇÃO DE TRAÇOS AUTÍSTICOS (ATA) \\ VALIDADE E CONFIABILIDADE DE UMA ESCALA PARA A DETECÇÃO DE CONDUTAS AUTÍSTICAS
}

\author{
FRANCISCO B. ASSUMPÇÃO JR * EVELYN KUCZYNSKI**, \\ MARCIA REGO GABRIEL ***, CRISTIANE CASTANHO ROCCA ****
}

\begin{abstract}
RESUMO - O objetivo deste estudo foi, a partir da análise de uma população de 31 pacientes portadores de deficiência mental e de 30 pacientes diagnosticados como portadores de autismo, conforme os critérios diagnósticos do DSM-IV, traduzir, adaptar e validar a escala de traços autísticos, construída em Barcelona por Ballabriga e colaboradores. O ponto de corte encontrado foi 15 ( $\mathrm{p}=0,05)$; o coeficiente de variação (confiabilidade), 0,27; a validade externa mostrou baixa concordância $(\mathrm{kappa}=0,04)$ e a validade interna foi $100 \%$, mostrando que, em todos os pacientes avaliados, os diagnósticos clínicos concordaram com os realizados através da escala. O índice de correlação foi 0,42 , mostrando-se específico para os quadros autísticos. Apresentou ainda, capacidade de discriminação e consistência interna, com alfa de Cronbach 0,71. Consideramos, assim, que a escala mostra-se confiável para a sua utilização em nosso meio.
\end{abstract}

PALAVRAS-CHAVE: autismo, diagnóstico, escala de avaliação.

\section{Validity and reliability of a scale for the assessment of austistic behaviour}

ABSTRACT - This study aimed to translate, to adapt and to validate the scale of autistic traits (ATA) developed by Ballabriga et al. in Barcelona, by the analysis of 31 patients with mental deficiency and 30 patients with autism, according to the DSM-IV criteria. The "cut off point" found was 15 ( $\mathrm{p}=0.05)$; the reliability coefficient was 0.27 ; the construct validity showed low agreement $(k a p p a=0.04)$ and the internal validity was $100 \%$, with all evaluated patients having their clinical diagnosis confirmed through the scale application. The correlation index was 0.42 , showing that it is specific for autism. The scale presents discrimination capacity and internal consistency, and the Cronbach's alpha was 0.71 . We consider that achieved results show that the scale is reliable to be used in our population.

KEY WORDS: autism, diagnostic, evaluation scale.

O autismo infantil é visto hoje como um transtorno de desenvolvimento caracterizado por incapacidade qualitativa na integração social, na comunicação verbal e não verbal, com repertório de atividades e interesses acentuadamente restritos, com início antes dos 3 anos de idade ${ }^{1}$. É considerado um transtorno predominantemente $\operatorname{cognitivo} o^{2-4}$, tendo como característica central a impossibilidade de compreensão do estado mental das demais pessoas conforme o referido por Hobson $^{5}$ e mais tarde bastante estudado por Baron-Cohen e col. ${ }^{6}$ que caracteriza os quadros autísticos como uma falha na, assim chamada, "teoria da mente", ou seja uma incapacidade em compreender os próprios estados mentais ou os de outras pessoas., embora o próprio Hobson ${ }^{5}$ refira o déficit

Estudo realizado no Serviço de Psiquiatria da Infância e da Adolescência do Instituto de Psiquiatria do Hospital das Clínicas (HC) da Faculdade de Medicina (FM) da Universidade de São Paulo (USP): *Professor Livre-Docente do Departamento de Psiquiatria da FMUSP; **Pediatra, Psiquiatra da Infância e da Adolescência, Pós Graduanda do Departamento de Psiquiatria da FMUSP; ***Psicóloga, Pós Graduanda do Programa de Neurociências da Faculdade de Psicologia da USP; ****Psicóloga, Pós Graduanda do Programa de Fisiologia Funcional da FMUSP.

Dr. Francisco B. Assumpção Jr. - Instituto de Psiquiatria do Hospital das Clínicas FMUSP - Av. Dr. Ovídio Pires de Campos s/n - 05403-900 São Paulo SP - Brasil. 
comunicacional como fator também importante nos quadros autísticos. Sua relação íntima com a deficiência mental também existe, de tal modo que Wing ${ }^{7}$ propõe um "continuum" no qual a variabilidade sintomatológica é decorrente do comprometimento intelectual, passando a ser de importância o rastreamento desses quadros de maneira a que não se superponham seus diagnósticos aos de retardo mental.

A identificação de autismo é assim, de fundamental importância, e as escalas de avaliação permitem mensurar as condutas apresentadas de maneira a se estabelecer um diagnóstico de maior confiabilidade. Apresentam-se estas sob a forma de questionários, de lista de sintomas ou de inventários ${ }^{8}$. Essas escalas, inspiradas nas ciências exatas, embora utilizadas algumas vezes de maneira indiscriminada, podem ser aplicadas na criança para avaliação de aspectos específicos do comportamento9. Dessa maneira, devem ser utilizadas para pesquisa ou para avaliação da evolução de determinados quadros. A escala de traços autísticos (ATA), elaborada por Ballabriga e col. ${ }^{10}$, nasceu a partir da discussão dos aspectos mais significativos da síndrome, partindo-se de diferentes instrumentos e da experiência clínica dos autores, sendo, entretanto, embasada primordialmente nos critérios diagnósticos do DSM III-R ${ }^{11}$. Isso porque a sua aparição unificou uma série de critérios, embora, conforme referem Volkmar e col. ${ }^{12}$, seja extremamente ampla e muito pouco específica, envolvendo uma série de quadros e mesmo de sinais que pertencem a um grupamento muito maior de quadros.

O objetivo deste estudo foi verificar a aplicabilidade da referida escala em nosso meio, adaptando-a aos atuais critérios do DSM IV. Esse embasamento, bem como sua fácil aplicabilidade, tornam-na passível de ser utilizada por pessoal não especializado com a finalidade de triar casos suspeitos de autismo, bem como por profissionais não médicos que tenham o intuito de avaliar o desenvolvimento da criança autista durante programas de reabilitação.

\section{MÉTODO}

A escala ATA é composta por 23 subescalas, cada uma das quais dividida em diferentes itens (Anexo I). Sua construção foi realizada levando-se em conta os critérios diagnósticos do DSM-III, DSM-III-R e da CID-10 e, na nossa padronização, foram utilizadas também as correções de critérios decorrentes da publicação do DSM$\mathrm{IV}^{1}$. Assim sendo, quinze das suas subescalas são representativas dos três critérios básicos e somente as subescalas 8,10 e 15 são apontadas como itens adicionais nas escalas ERCN ("Échelle d'évaluation resumée du comportement autistique"), ERCA-IIIA (“Échelle d'évaluation resumée du comportement du nourrison") e na escala de Riviére. As subescalas 1, 16 e 21 são aquelas que se apresentam com maior frequência nos instrumentos diagnósticos revisados pelos autores.

Assim sendo, a partir de sua construção, pode-se observar que a escala ATA é um instrumento de fácil aplicação, acessível a profissionais que têm contato direto com a população autista, por exemplo, professores, bem como pais, informando o estado atual do paciente. Ela é aplicada por profissional conhecedor do quadro, embora não necessariamente médico, sendo ele o responsável pela avaliação das respostas dadas, em função de cada item. Não é portanto uma entrevista diagnóstica, mas uma prova estandardizada que dá o perfil conductual da criança, embasada nos diferentes aspectos diagnósticos. Baseia-se na observação e permite fazer seguimentos longitudinais da evolução, tendo por base a sintomatologia autística, auxiliando também a elaboração de um diagnóstico mais confiável desses quadros. É administrada após informação detalhada dos dados clínicos e evolutivos da criança, podendo auxiliar no processo terapêutico, possibilitando a avaliação constante. Pode ser aplicada a partir dos dois anos de idade e, mesmo considerando-se que apresenta muitos itens específicos, os autores referem tempo pequeno para sua aplicação, ao redor de 20 a 25 minutos. Na aplicação em nossa população, o tempo médio ficou entre 20 e 30 minutos, aproximadamente.

A escala se pontua com base nos seguintes critérios: cada subescala da prova tem um valor de 0 a 2 ; pontua-se a escala positiva no momento em que um dos itens for positivo; a pontuação global da escala se faz a partir da soma aritmética de todos os valores positivos da subescala.

Para a adaptação ao nosso meio, o questionário foi traduzido do espanhol para o português, com posterior correção realizada por profissional competente na área e na língua de origem do questionário. Posteriormente, foi aplicado em 30 crianças diagnosticadas como portadoras de síndrome autística, provenientes do Serviço de Psiquiatria da Infância e da Adolescência do Instituto de Psiquiatria do HC/FMUSP e diagnosticadas por diferentes profissionais, a partir dos critérios diagnósticos do DSM-IV ${ }^{1}$. Foi aplicado também em 31 crianças portadoras de deficiência mental em grau moderado $(36<\mathrm{Q}<50)$, provenientes do Centro de Habilitação da APAE-SP e 


\section{ANEXO 1. ESCALA DE TRAÇOS AUTÍSTICOS.}

1. DIFICULDADE NA INTERAÇÃO SOCIAL - O desvio da sociabilidade pode oscilar entre formas leves como, por exemplo, um certo negativismo e a não aceitação do contato ocular, até formas mais graves, como um intenso isolamento - 1. Não sorri; 2. Ausência de aproximações espontâneas; 3. Não busca companhia; 4. Busca constantemente seu cantinho (esconderijo); 5. Evita pessoas; 6. É incapaz de manter um intercâmbio social; 7. Isolamento intenso.

II. MANIPULAÇÃO DO AMBIENTE - O problema da manipulação do ambiente pode apresentar-se a nível mais ou menos grave, como, por exemplo, não responder às solicitações e manter-se indiferente ao ambiente. $O$ fato mais comum é a manifestação brusca de crises de birra passageira, risos incontroláveis e sem motivo, tudo isso com o fim de conseguir ser o centro da atenção - 1. Não responde às solicitações; 2. Mudança repentina de humor; 3. Mantém-se indiferente, sem expressão; 4. Risos compulsivos; 5. Birra e raiva passageira; 6. Excitação motora ou verbal (ir de um lugar a outro, falar sem parar).

III. UTILIZAÇÃO DAS PESSOAS A SEU REDOR - A relação que mantém com o adulto quase nunca é interativa, dado que normalmente utiliza-se do adulto como o meio para conseguir o que deseja - 1. Utiliza-se do adulto como um objeto, levando-o até aquilo que ele deseja; 2 . $O$ adulto lhe serve como apoio para conseguir o que deseja (p.ex.: utiliza o adulto como apoio para pegar bolacha); 3. O adulto é o meio para suprir uma necessidade que não é capaz de realizar só (p.ex.: amarrar sapatos); 4. Se o adulto não responde as suas demandas, atua interferindo na conduta desse adulto.

IV. RESISTENNCIA À MUDANÇA - A resistência à mudança pode variar da irritabilidade até a franca recusa - 1 . Insistente em manter a rotina; 2. Grande dificuldade em aceitar fatos que alteram sua rotina, tais como mudanças de lugar, de vestuário e na alimentação; 3. Apresenta resistência a mudanças, persistindo na mesma resposta ou atividade.

V. BUSCA DE UMA ORDEM RÍGIDA - Manifesta tendência a ordenar tudo, podendo chegar a uma conduta de ordem obsessiva, sem a qual não consegue desenvolver nenhuma atividade - 1. Ordenação dos objetos de acordo com critérios próprios e pré-estabelecidos; 2. Prende-se a uma ordenação espacial (Cada coisa sempre em seu lugar); 3. Prende-se a uma sequência temporal (Cada coisa em seu tempo); 4. Prende-se a uma correspondência pessoa-lugar (Cada pessoa sempre no lugar determinado).

VI. FALTA DE CONTATO VISUAL. OLHAR INDEFENIDO - A falta de contato pode variar desde um olhar estranho até o constante evitar dos estímulos visuais - 1. Desvia os olhares diretos, não olhando nos olhos; 2. Volta a cabeça ou o olhar quando é chamado (olhar para fora); 3. Expressão do olhar vazio e sem vida; 4. Quando segue os estímulos com os olhos, somente o faz de maneira intermitente; 5. Fixa os objetos com uma olhada periférica, não central; 6. Dá a sensação de que não olha.

VII. MÍMICA INEXPRESSIVA - A inexpressividade mímica revela a carência da comunicação não verbal. Pode apresentar desde uma certa expressividade até uma ausência total de resposta - 1. Se fala, não utiliza a expressão facial, gestual ou vocal com a freqüência esperada; 2. Não mostra uma reação antecipatória; 3. Não expressa através da mímica ou olhar aquilo que quer ou o que sente; 4. Imobilidade facial.

VIII. DISTÚRBIOS DE SONO - Quando pequeno dorme muitas horas e, quando maior, dorme poucas horas, se comparado ao padrão esperado para a idade. Esta conduta pode ser constante, ou não - 1. Não quer ir dormir; 2. Se levanta muito cedo; 3. Sono irregular (em intervalos); 4. Troca ou dia pela noite; 5. Dorme muito poucas horas.

IX. ALTERAÇÃO NA ALIMENTAÇÃo - Pode ser quantitativa elou qualitativa. Pode incluir situações, desde aquela em que a criança deixa de se alimentar até aquela em que se opõe ativamente - 1. Seletividade alimentar rígida (ex.: come o mesmo tipo de alimento sempre); 2.Come outras coisas além de alimentos (papel, insetos); 3.Quando pequeno não mastigava; 4.Apresenta uma atividade ruminante; 5.Vômitos; 6.Come grosseiramente, esparrama a comida ou a atira; 7.Rituais ( esfarela alimentos antes da ingestão); 8.Ausência da paladar (Falta de sensibilidade gustativa).

X. DIFICULDADE NO CONTROLE DOS ESFÍNCTERES - O controle dos esfincteres pode existir, porém a sua utilização pode ser uma forma de manipular ou chamar a atenção do adulto - 1. Medo de sentar-se no vaso sanitário; 2. Utiliza os esfíncteres para manipular o adulto; 3. Utiliza os esfíncteres como estimulação corporal, para obtenção de prazer; 4. Tem controle diurno, porém o noturno é tardio ou ausente.

XI. EXPLORAÇÃO DOS OBJETOS (APALPAR, CHUPAR) - Analisa os objetos sensorialmente, requisitando mais os outros órgãos dos sentidos em detrimento da visão, porém sem uma finalidade específica - 1. Morde e 
engole objetos não alimentares; 2. Chupa e coloca as coisas na boca; 3. Cheira tudo; 4. Apalpa tudo. Examina as superfícies com os dedos de uma maneira minuciosa.

XII. USO INAPROPRIADO DOS OBJETOS - Não utiliza os objetos de modo funcional, mas sim de uma forma bizarra - 1. Ignora os objetos ou mostra um interesse momentâneo; 2. Pega, golpeia ou simplesmente os atira no chão; 3. Conduta atípica com os objetos (segura indiferentemente nas mãos ou gira); 4. Carrega insistentemente consigo determinado objeto; 5. Se interessa somente por uma parte do objeto ou do brinquedo; 6. Coleciona objetos estranhos; 7. Utiliza os objetos de forma particular e inadequada.

XIII. FALTA DE ATENÇÃO - Dificuldades na fixação e concentração. Às vezes, fixa a atenção em suas próprias produções sonoras ou motoras, dando a sensação de que se encontra ausente - 1. Quando realiza uma atividade, fixa a atenção por curto espaço de tempo ou é incapaz de fixá-la; 2. Age como se fosse surdo; 3. Tempo de latência de resposta aumentado; 4. Entende as instruções com dificuldade (quando não lhe interessa, não as entende); 5. Resposta retardada; 6. Muitas vezes dá a sensação de ausência.

XIV. AUSÊNCIA DE INTERESSE PELA APRENDIZAGEM - Não tem nenhum interesse por aprender, buscando solução nos demais. Aprender representa um esforço de atenção e de intercâmbio pessoal, é uma ruptura em sua rotina - 1. Não quer aprender; 2. Se cansa muito depressa, ainda que em atividade que goste; 3. Esquece rapidamente; 4. Insiste em ser ajudado, ainda que saiba fazer; 5. Insiste constantemente em mudar de atividade.

XV. FALTA DE INICIATIVA - Busca constantemente a comodidade e espera que lhe dêem tudo pronto. Não realiza nenhuma atividade funcional por iniciativa própria - 1. É incapaz de ter iniciativa própria; 2. Busca a comodidade; 3. Passividade, falta de interesse; 4. Lentidão; 5. Prefere que outro faça o trabalho para ele.

XVI. ALTERAÇÃO DE LINGUAGEM E COMUNICAÇÃO - É uma característica fundamental do autismo, que pode variar desde um atraso de linguagem até formas mais severas, com uso exclusivo de fala particular e estranha - 1. Mutismo; 2. Estereotipias vocais; 3. Entonação incorreta; 4. Ecolalia imediata elou retardada; 5. Repetição de palavras ou frases que podem ou não ter valor comunicativo; 6. Emite sons estereotipados quando está agitado e em outras ocasiões, sem nenhuma razão aparente; 7. Não se comunica por gestos; 8 . As interações com adulto não são nunca um diálogo.

XVII. NÃO MANIFESTA HABILIDADES E CONHECIMENTOS - Nunca manifesta tudo aquilo que é capaz de fazer ou agir, no que faz referência a seus conhecimentos e habilidades, dificultando a avaliação dos profissionais - 1. Ainda que saiba fazer uma coisa, não a realiza, se não quiser; 2. Não demonstra o que sabe até que tenha uma necessidade primária ou um interesse iminentemente específico; 3. Aprende coisas, porém somente a demonstra em determinados lugares e com determinadas pessoas; 4. As vezes surpreende por suas habilidades inesperadas.

XVIII. REAÇÕES INAPROPRIADAS ANTE A FRUSTRAÇÃO - Manifesta desde o aborrecimento à reação de cólera, ante a frustração - 1. Reações de desagrado caso seja esquecida alguma coisa; 2. Reações de desagrado caso seja interrompida alguma atividade que goste; 3. Desgostoso quando os desejos e as expectativas não se cumprem; 4. Reações de birra.

XIX. NÃO ASSUME RESPONSABILIDADES - Por princípio, é incapaz de fazer-se responsável, necessitando de ordens sucessivas para realizar algo - 1. Não assume nenhuma responsabilidade, por menor que seja; 2. Para chegar a fazer alguma coisa, há que se repetir muitas vezes ou elevar o tom de voz.

XX. HIPERATIVIDADE/ HIPOATIVIDADE - A criança pode apresentar desde agitação, excitação desordenada e incontrolada, até grande passividade, com ausência total de resposta. Estes comportamentos não tem nenhuma finalidade - 1. A criança está constantemente em movimento; 2. Mesmo estimulada, não se move; 3. Barulhento. Dá a sensação de que é obrigado a fazer ruído/barulho; 4. Vai de um lugar a outro, sem parar; 5. Fica pulando (saltando) no mesmo lugar; 6. Não se move nunca do lugar onde está sentado.

XXI. MOVIMENTOS ESTEREOTIPADOS E REPETITIVOS - Ocorrem em situações de repouso ou atividade, com início repentino - 1. Balanceia-se; 2. Olha e brinca com as mãos e os dedos; 3. Tapa os olhos e as orelhas; 4. Dá pontapés; 5. Faz caretas e movimentos estranhos com a face; 6. Roda objetos ou sobre si mesmo; 7. Caminha na ponta dos pés ou saltando, arrasta os pés, anda fazendo movimentos estranhos; 8. Torce o corpo, mantém uma postura desequilibrada, pernas dobradas, cabeça recolhida aos pés, extensões violentas do corpo.

XXII. IGNORA O PERIGO - Expõe-se sem ter consciência do perigo - 1. Não se dá conta do perigo; 2. Sobe em todos os lugares; 3. Parece insensível a dor. 
diagnosticadas por equipe multidisciplinar composta por pediatra, neurologista, psiquiatra, psicólogo e assistente social. Ambas as populações eram similares sob o ponto de vista sócio-econômico.

Sabendo-se que a incidência do fenômeno na população é de 0,0004 , podemos calcular a estimativa do tamanho da amostra com intervalo de confiança de $95 \%$ e erro máximo de 2 unidades para uma população de 75000 pessoas. Esse número corresponde a 29 e assim, a mostra de 30 é representativa, uma vez que para 30 elementos teremos um erro máximo de 1,3 unidades.

Os questionários foram aplicados por duas psicólogas e ambas as populações apresentavam idades entre 2 e 18 anos. Os dados obtidos foram estudados mediante análise da variância de cada um dos itens, variância total e alfa de Cronbach.

\section{RESULTADOS}

A média do total de pontos obtidos foi $15,76( \pm 8,61)$ para a população portadora de deficiência mental e $31,56( \pm 8,32)$ para a população autista. O ponto de corte encontrado foi 15 , para $p=0,05$, com erro padrão de 1,5 e coeficiente de variação (confiabilidade) de 0,27 . Em relação à validade externa, podemos observar que a concordância obtida entre os critérios do DSM-IV é baixo, com kappa da ordem de 0,04 . Na análise da consistência interna da escala utilizando o coeficiente alfa de Cronbach, obtendo-se o valor 0,71, que pode ser considerado alto (Tabelas 1, 2 e 3).

Tabela 1. Estudo da população autista através da ATA. Dados clínicos; média de pontos = 31,56.

\begin{tabular}{|c|c|c|c|c|}
\hline Caso & Sexo & Idade (anos) & "Escore" médio associado & Quadro clínico \\
\hline 1 & $\mathrm{M}$ & 16 & 33 & \\
\hline 2 & M & 14 & 39 & \\
\hline 3 & M & 14 & 37 & \\
\hline 4 & $\mathrm{~F}$ & 8 & 40 & Toxoplasmose \\
\hline 5 & M & 12 & 41 & \\
\hline 6 & M & 7 & 29 & Fra-X \\
\hline 7 & $\mathrm{~F}$ & 16 & 18 & \\
\hline 8 & M & 19 & 40 & \\
\hline 9 & M & 13 & 29 & \\
\hline 10 & M & 7 & 35 & \\
\hline 11 & M & 4 & 27 & \\
\hline 12 & M & 13 & 40 & \\
\hline 13 & M & 14 & 41 & Síndrome de Aarskog \\
\hline 14 & $\mathrm{~F}$ & 8 & 41 & \\
\hline 15 & M & 6 & 41 & \\
\hline 16 & M & 10 & 15 & \\
\hline 17 & M & 10 & 25 & Fra-X \\
\hline 18 & M & 11 & 27 & \\
\hline 19 & M & 10 & 33 & \\
\hline 20 & M & 6 & 38 & \\
\hline 21 & M & 16 & 31 & Fra-X \\
\hline 22 & M & 2 & 15 & \\
\hline 23 & M & 5 & 32 & \\
\hline 24 & $\mathrm{~F}$ & 17 & 18 & \\
\hline 25 & M & 7 & 25 & Síndrome de Aarskog \\
\hline 26 & M & 11 & 29 & \\
\hline 27 & $\mathrm{~F}$ & 9 & 37 & \\
\hline 28 & M & 10 & 32 & \\
\hline 29 & M & 16 & 40 & \\
\hline 30 & M & 7 & 20 & \\
\hline
\end{tabular}


Tabela 2. Estudo da população deficiente mental através da ATA. Dados clínicos; média de pontos = 15,76.

\begin{tabular}{|c|c|c|c|c|}
\hline Caso & Sexo & Idade (anos) & "Escore" médio associado & Quadro clínico \\
\hline 1 & M & 16 & 20 & \\
\hline 2 & M & 9 & 14 & Síndrome de Down \\
\hline 3 & $\mathrm{~F}$ & 15 & 20 & \\
\hline 4 & M & 15 & 8 & \\
\hline 5 & $\mathrm{~F}$ & 11 & 22 & \\
\hline 6 & M & 8 & 24 & Síndrome de Prader-Willie \\
\hline 7 & $\mathrm{~F}$ & 8 & 17 & Hipotiroidismo \\
\hline 8 & M & 17 & 22 & \\
\hline 9 & $\mathrm{~F}$ & 14 & 16 & \\
\hline 10 & $\mathrm{~F}$ & 5 & 11 & Fenilcetonúria \\
\hline 11 & M & 8 & 35 & \\
\hline 12 & M & 11 & 34 & Síndrome de Klippel-Trenaunay \\
\hline 13 & $\mathrm{~F}$ & 6 & 19 & \\
\hline 14 & M & 10 & 20 & Fra-X \\
\hline 15 & M & 8 & 27 & \\
\hline 16 & M & 9 & 14 & \\
\hline 17 & $\mathrm{~F}$ & 13 & 6 & \\
\hline 18 & M & 12 & 25 & \\
\hline 19 & M & 15 & 4 & Síndrome de Down \\
\hline 20 & M & 14 & 26 & \\
\hline 21 & M & 5 & 15 & \\
\hline 22 & $\mathrm{~F}$ & 11 & 31 & Síndrome de Aarskog \\
\hline 23 & M & 7 & 7 & Fenilcetonúria \\
\hline 24 & $\mathrm{~F}$ & 11 & 15 & Fra-X \\
\hline 25 & $\mathrm{~F}$ & 4 & 18 & \\
\hline 26 & $\mathrm{~F}$ & 11 & 4 & \\
\hline 27 & M & 10 & 2 & \\
\hline 28 & M & 14 & 6 & \\
\hline 29 & M & 6 & 8 & XXXY \\
\hline 30 & M & 14 & 4 & \\
\hline 31 & $\mathrm{~F}$ & 14 & 14 & \\
\hline
\end{tabular}

Cinco itens $(5,7,8,13$ e 23) apresentaram baixa correlação com os demais, embora somente o de número 23 tenha se mostrado negativo. A validade interna encontrada foi $100 \%$, uma vez que todos os pacientes diagnosticados clinicamente concordaram com o diagnóstico apresentado pela escala em questão.

Na comparação entre as amostras de pacientes autistas e de pacientes portadores de deficiência mental, encontramos média de 15,76 pontos para portadores de deficiência mental contra média de 31,56 para os portadores de autismo, mostrando assim índice de correlação de 0,42 , o que denota a especificidade da observação em relação aos quadros autísticos.

\section{DISCUSSÃO}

A avaliação de comportamentos que permita o reconhecimento de traços autísticos e, em consequência, possibilite um diagnóstico populacional é de extrema importância uma vez que muitos dos portadores do quadro misturam-se com a população deficiente mental, ficando restrita a esse atendimento e não sendo passíveis de identificação.

A proposta deste estudo foi a de validar a escala de traços autísticos, desenvolvida em Barcelona, adaptando-a ao DSM-IV e à realidade brasileira, uma vez que ela permite avaliar, de maneira 
Tabela 3. Correlação da análise de cada um dos itens da escala ATA, em que cada valor mostra a correlação entre cada item e a soma dos itens restantes.

\begin{tabular}{cc}
\hline Item & Valor \\
\hline 1 & 0,47 \\
2 & 0,39 \\
3 & 0,74 \\
4 & 0,44 \\
5 & 0,15 \\
6 & 0,47 \\
7 & 0,15 \\
8 & 0,23 \\
9 & 0,81 \\
10 & 0,28 \\
11 & 0,73 \\
12 & 0,66 \\
13 & 0,16 \\
14 & 0,56 \\
15 & 0,51 \\
16 & 0,31 \\
17 & 0,45 \\
18 & 0,31 \\
19 & 0,72 \\
20 & 0,55 \\
21 & 0,24 \\
22 & 0,39 \\
23 & 0,31 \\
\hline
\end{tabular}

confiável, as condutas autistas na criança. O ponto de corte obtido (15), para $\mathrm{p}=0,05$ e coeficiente de variação de 0,27 , permite-nos estabelecer uma suspeita diagnóstica bastante precisa do quadro em questão. Esse dado pode ser utilizado com a finalidade de um primeiro diagnóstico a ser realizado em grandes populações nas quais a suspeita de autismo confunde-se com quadros de retardo mental ou de outras patologias psiquiátricas. A diferença observada por nós, nos escores obtidos em ambas as populações estudadas, mostra sua sensibilidade para a detecção de quadros suspeitos. Assim, embora não dispense o diagnóstico clínico, este instrumento permite uma primeira triagem desses quadros e, posteriormente, pode ser utilizado também como guia da evolução do tratamento, pois engloba as diferentes áreas comprometidas pela patologia..

Apresenta grande sensibilidade $(0,96) \mathrm{em}$ relação aos critérios do DSM-IV, para os quais foi adaptada, considerando-se que em sua construção foram utilizados os critérios do DSMIII e do DSM-III-R Embora não substitua nem proponha critérios diferentes daqueles utilizados pelos atuais sistemas classificatórios, pois é baseada em um deles (DSM-IV), acreditamos que sua utilização se mostre de grande valia para o estudo do autismo em nosso meio, visto que pode ser aplicada por profissionais treinados em seu uso mesmo que não tenham formação psiquiátrica.

\section{REFERÊNCIAS}

1. APA. Manual diagnóstico e estatístico de transtornos mentais - DSM-IV. Porto Alegre: Artes Médicas, 1995.

2. Baron-Cohen S. Social and pragmatic deficits in autism: cognitive or affective? J Autism Dev Disord 1988;18:379-401.

3. Baron-Cohen S. Autism, a specific cognitive disorder 'mind - blindness'. Int Rev Psychiat 1990;2:81-90, 1990.

4. Baron-Cohen S. The development of a theory of mind in autism: deviance and delay? Psychiat Clin N Am 1991;14:33-52.

5. Hobson RP. What is autism? Psychiat Clin N Amer 1991;14:1-18.

6. Baron-Cohen S, Leslie AM, Frith U. Does the autistic child have a theory of mind? Cognition 1985;21:37-46.

7. Wing L. The autistic continuum. In Wing L. Aspects of autism: biological research. London: Royal College of Psychiatrists \& The National Autistic Society, 1988.

8. Barthélémy C. Évaluations cliniques quantitatives em pédopsychiatrie. Neuropsychiatrie de l'Enfance 1986;34:636-691.

9. Lelord GF. Remarques sur les échelles d'évaluation. Neuropsychiatrie de l'Enfance 1986;34:119-121.

10. Ballabriga MCJ, Escudé RMC, Llaberia ED. Escala d'avaluació dels trests autistes (A.T.A.): validez y fiabilidad de una escala para el examen de las conductas autistas. Rev Psiquiatria Infanto-Juvenil 1994;4:254-263.

11. APA, Manual diagnóstico e estatístico de transtornos mentais - DSM-III-R. São Paulo: Manole, 1989.

12. Volkmar FR, Bergman J, Cohen DJ, Ciccheti DN. DSM-III and DSM III-R diagnosis of autism. Am J Psychiatry 1988;145:1404-1408. 